\title{
Vital Signs: Preventing Antibiotic-Resistant Infections in Hospitals - United States, 2014
}

Lindsey M. Weiner, $\mathrm{MPH}^{1}$; Scott K. Fridkin, $\mathrm{MD}^{1}$; Zuleika Aponte-Torres, $\mathrm{MPH}^{1}$; Lacey Avery, $\mathrm{MA}^{1}$; Nicole Coffin, MA¹; Margaret A. Dudeck, MPH ${ }^{1}$; Jonathan R. Edwards, MStat ${ }^{1}$; John A. Jernigan, MD $^{1}$; Rebecca Konnor, MPH ${ }^{1}$; Minn M. Soe, MBBS, MPH ${ }^{1}$; Kelly Peterson ${ }^{1}$; L. Clifford McDonald, MD ${ }^{1}$

On March 3, 2016, this report was posted as an MMWR Early Release on the MMWR website (http://www.cdc.gov/mmwr).

\section{Abstract}

Background: Health care-associated antibiotic-resistant (AR) infections increase patient morbidity and mortality and might be impossible to successfully treat with any antibiotic. CDC assessed health care-associated infections (HAI), including Clostridium difficile infections (CDI), and the role of six AR bacteria of highest concern nationwide in several types of health care facilities.

Methods: During 2014, approximately 4,000 short-term acute care hospitals, 501 long-term acute care hospitals, and 1,135 inpatient rehabilitation facilities in all 50 states reported data on specific infections to the National Healthcare Safety Network. National standardized infection ratios and their percentage reduction from a baseline year for each HAI type, by facility type, were calculated. The proportions of AR pathogens and HAIs caused by any of six resistant bacteria highlighted by CDC in 2013 as urgent or serious threats were determined.

Results: In 2014, the reductions in incidence in short-term acute care hospitals and long-term acute care hospitals were $50 \%$ and $9 \%$, respectively, for central line-associated bloodstream infection; 0\% (short-term acute care hospitals), 11\% (long-term acute care hospitals), and 14\% (inpatient rehabilitation facilities) for catheter-associated urinary tract infection; 17\% (short-term acute care hospitals) for surgical site infection, and 8\% (short-term acute care hospitals) for CDI. Combining HAIs other than CDI across all settings, $47.9 \%$ of Staphylococcus aureus isolates were methicillin resistant, $29.5 \%$ of enterococci were vancomycin-resistant, $17.8 \%$ of Enterobacteriaceae were extended-spectrum beta-lactamase phenotype, 3.6\% of Enterobacteriaceae were carbapenem resistant, $15.9 \%$ of Pseudomonas aeruginosa isolates were multidrug resistant, and $52.6 \%$ of Acinetobacter species were multidrug resistant. The likelihood of HAIs caused by any of the six resistant bacteria ranged from $12 \%$ in inpatient rehabilitation facilities to $29 \%$ in long-term acute care hospitals.

Conclusions: Although there has been considerable progress in preventing some HAIs, many remaining infections could be prevented with implementation of existing recommended practices. Depending upon the setting, more than one in four of HAIs excluding CDI are caused by AR bacteria.

Implications for Public Health Practice: Physicians, nurses, and health care leaders need to consistently and comprehensively follow all recommendations to prevent catheter- and procedure-related infections and reduce the impact of AR bacteria through antimicrobial stewardship and measures to prevent spread.

\section{Introduction}

Antibiotic-resistant (AR) bacteria are a worldwide public health threat. A 2013 CDC report outlined the top 18 urgent, serious, and concerning AR threats in the United States (1). Among the 15 urgent and serious threats, seven are bacteria predominately acquired during health care. Clostridium difficile is included among these; although $C$. difficile is not drugresistant, the infections it causes and its spread are exacerbated by inappropriate antibiotic use and inadequate infection control, similar to the six other AR bacteria. Preventing health care-associated infections (HAIs) provides immediate benefit in reducing the impact of antibiotic resistance on human health. When combined with antibiotic stewardship and steps to prevent transmission as outlined in the National Action Plan to Combat Antibiotic Resistant Bacteria (2), preventing HAIs is critical to reducing the public health threat of AR bacteria.

More than half of hospitalized patients are receiving antibiotics on any given day (3), and about one in 25 have one or more HAIs (4). During 2011 an estimated 722,000 HAIs occurred in U.S acute care hospitals, and approximately 75,000 patients with HAIs died during hospitalization (4). More than half of these HAIs include $C$. difficile infections (CDIs), urinary tract infections, bloodstream infections, or surgical site infections (SSIs). The HAI National Action Plan (5) calls for CDC to monitor progress toward established goals through 
the National Healthcare Safety Network (NHSN). This report describes progress toward reducing HAIs in the United States and describes the frequency of six AR bacteria of urgent or serious public health concern among reported HAIs in 2014.

\section{Methods}

HAI data on central line-associated bloodstream infections (CLABSIs), catheter-associated urinary tract infections (CAUTIs), surgical site infections (SSIs), and laboratoryidentified CDI events for 2014 were reported to NHSN from hospitals in all 50 states, the District of Columbia, and Puerto Rico, using standard NHSN definitions (6-8). Data are presented separately for acute care hospitals (including critical access hospitals), long-term acute care hospitals, and inpatient rehabilitation facilities, because reporting timelines and type of HAIs reported varied among the different settings.

Standardized infection ratios (SIRs), a statistic used to track HAIs over time, were used to compare the observed number of infections reported during 2014 with the predicted number of infections, based on national aggregate data reported during a historical baseline time period. SIRs for different infections were adjusted for key risk factors (9-11). Baseline time periods among short-term acute care hospitals were 2006-2008 for CLABSIs and SSIs, 2009 for CAUTIs, and 2010-2011 for CDIs. Among long-term acute care hospitals and inpatient rehabilitation facilities the baseline time period was 2013 for both CLABSIs and CAUTIs. The SSI data include 10 procedures that approximate procedures included in the Centers for Medicare and Medicaid Services Surgical Care Improvement Project and were performed during 2014 (10).

Pathogen and susceptibility data are provided by the facility's designated clinical microbiology laboratory. No more than three pathogens per HAI could be reported. Susceptibility results for each pathogen were reported as "susceptible," "intermediate," "resistant," or "not tested" (12). The six AR phenotypes included the urgent threat of carbapenemresistant Enterobacteriaceae, along with the serious threats of methicillin-resistant Staphylococcus aureus, vancomycinresistant enterococci, extended-spectrum beta-lactamase phenotype Enterobacteriaceae, multidrug-resistant (MDR) P. aeruginosa, and MDR Acinetobacter species. The criteria used to define each phenotype approximated interim standard definitions for defining multidrug resistance as used in the CDC AR Threat Report (1), along with updated criteria for carbapenem-resistant Enterobacteriaceae (13).

A pooled mean percentage of resistant pathogens, based on the sum of pathogens that tested resistant, divided by the sum of pathogens tested, was calculated for each threat pathogen by HAI type and facility type. In addition, the likelihood that an HAI was associated with any of the six antibiotic-resistant threat pathogens was calculated as the sum of HAIs with any resistant phenotype divided by the sum of HAIs reported (regardless of whether another pathogen or, in the case of SSI, no pathogen was reported).

\section{Results}

In 2014, approximately 4,000 acute care hospitals $(3,655$ reported CLABSI data, 3,791 reported data on CAUTI, 3,994 reported CDI, and 3,618 reported SSI), 501 longterm acute care hospitals, and 1,135 inpatient rehabilitation facilities contributed data. Within acute care hospitals, 17,758 CLABSIs, 35,760 CAUTIs, 101,074 hospital-onset CDIs, and 15,927 SSIs from selected procedures were reported. The corresponding SIRs (and 95\% confidence intervals) were 0.495 (0.488-0.502) for CLABSI, 1.00 (0.990-1.010) for CAUTI, 0.924 (0.918-0.929) for CDI, and 0.827 (0.815-0.840) for SSI, corresponding to percentage decreases compared with the historical baseline assessment ranging from $0 \%$ (CAUTI) to 50\% (CLABSI) (Figure). The percentage change from 2013 to 2014 was $-8 \%$ for CLABSI, $-5 \%$ CAUTI, $+4 \%$ for CDI, and $+2 \%$ for SSI.

Among long-term acute care hospitals, 2,928 CLABSIs and 4,467 CAUTIs were reported; after risk adjustment, the SIRs were 0.909 (0.876-0.942) for CLABSI and 0.893 (0.867-0.920) for CAUTI, corresponding to $9 \%$ and $11 \%$ decreases, respectively, compared with baseline. Within inpatient rehabilitation facilities, 1,449 CAUTIs were reported, for an SIR of $0.856(0.813-0.901)$ or a $14 \%$ reduction compared with baseline.

Combining HAIs across all settings, $47.9 \%$ of $S$. aureus infections were resistant to methicillin, $29.5 \%$ of enterococci were resistant to vancomycin, $17.8 \%$ of Enterobacteriaceae were extended-spectrum beta-lactamase phenotype, 3.6\% of Enterobacteriaceae were carbapenem-resistant, $15.9 \%$ of P. aeruginosa, and 52.6\% of Acinetobacter species were MDR. Notably, the percentage resistance varied by facility type and was consistently higher in long-term acute care hospitals (Table).

During 2014, the likelihood of any of the six AR threat bacteria varied by HAI type and facility type. Overall, among short-term acute care hospitals, $14 \%$ of all HAIs were caused by one of the six AR threat bacteria, including $18 \%$ of CLABSIs (3,348 of 18,373), $15 \%$ of SSIs $(2,583$ of 17,512$), 10 \%$ of CAUTIs $(3,601$ of 34,621$)$. Among long-term acute care hospitals, $28 \%$ of CLABSIs ( 808 of 2,873 ) and $29 \%$ of CAUTIs $(1,251$ of 4,293) were caused by one of these organisms, and among inpatient rehabilitation facilities, $12 \%$ of CAUTIs (164 of 1,349$)$ were caused by one of these six bacteria. Pooled over all facility types, $14.9 \%$ of the 79,021 HAIs reported were associated with one of the AR threat pathogens. 
FIGURE. Standardized infection ratios (SIRs) with 95\% confidence intervals for health care-associated infections (HAIs) reported from acute care hospitals as a measure of prevention progress compared with the baseline year,* by HAI type and year - National Healthcare Safety Network, United States, 2008-2014

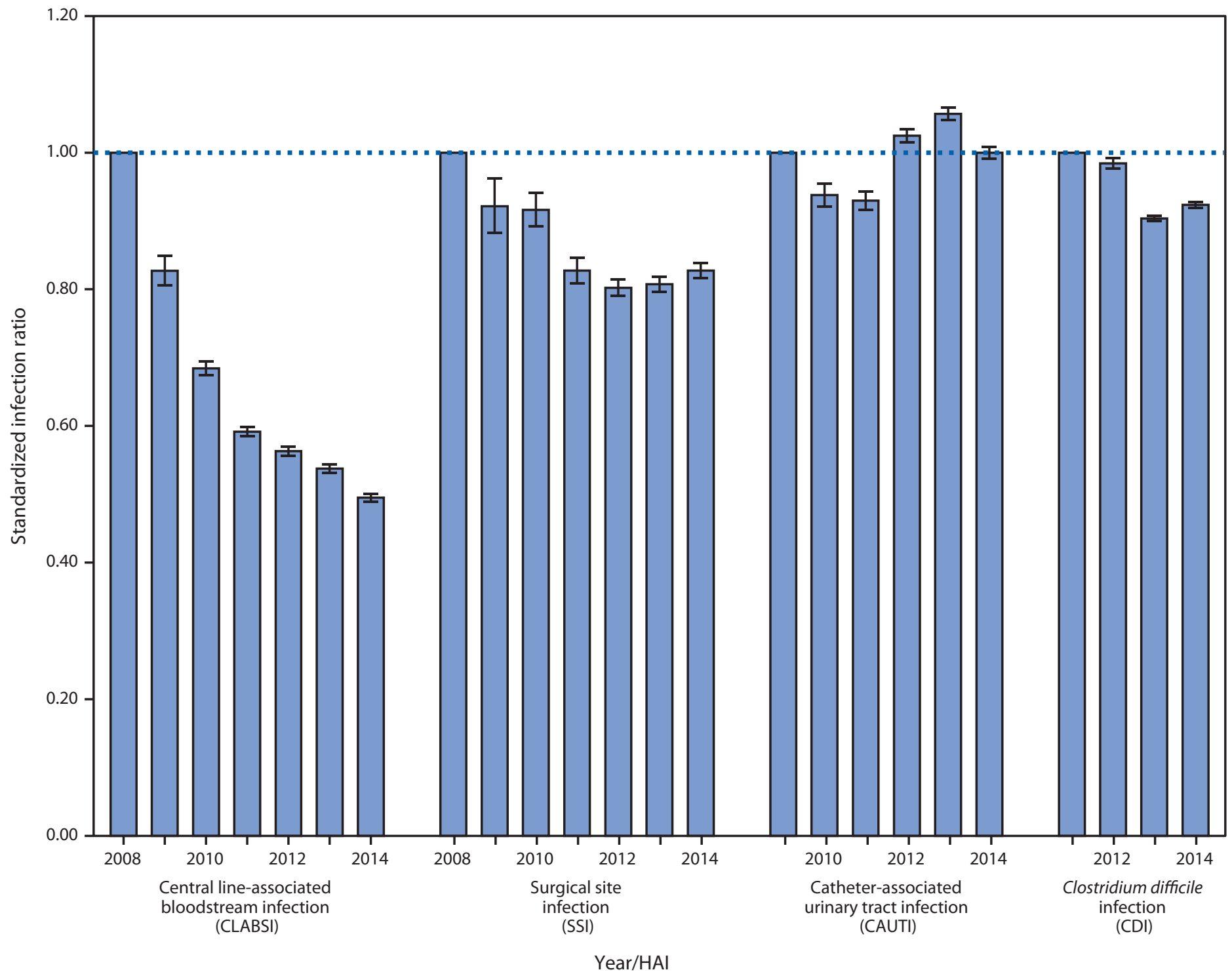

* First year with calculated SIR value, 1.00. Baseline periods were 2006-2008 for CLABSI and SSI, 2009 for CAUTI, and 2010-2011 for CDI. Data were calculated using the latest year of the baseline period.

+ SSI data are inclusive of the 10 selected procedures that approximate the procedures included in the Centers for Medicare \& Medicaid Services Surgical Care Improvement Project and were performed during 2014.

$\S$ Device-associated infections include both intensive care unit (ICU) and non-ICU locations. http://www.cdc.gov/hai/surveillance/nhsn_nationalreports.html.

\section{Conclusions and Comment}

In the United States, approximately 2 million persons become ill every year with AR infections, and approximately 23,000 die. This report is the first to combine national data on AR bacteria threats with progress on HAI prevention. In 2014, the incidence of CLABSI in acute care hospitals reached the 2013 goal established by the HAI Action Plan (5), decreasing
50\% during 2008-2014. This is important given the high morbidity, mortality, and excess costs associated with CLABSIs $(14,15)$, which are partially related to the frequency with which methicillin-resistant Staphylococcus aureus, vancomycinresistant enterococci, and extended-spectrum beta-lactamase phenotype Enterobacteriaceae cause these infections (Table). In addition, CAUTIs in acute care hospitals decreased overall by 5\% during 2013-2014 and, although not quantified in 
TABLE. Pooled mean percentage of tested isolates of six urgent or serious antibiotic-resistant threat pathogens that were antibiotic-resistant, by type of health care facility and type of health care-associated infection reported - National Healthcare Safety Network, United States, 2008-2014*

\begin{tabular}{|c|c|c|c|c|c|c|c|c|}
\hline \multirow[b]{3}{*}{ Facility type/Antibiotic-resistant threat pathogen } & \multicolumn{8}{|c|}{ Health care-associated infection type } \\
\hline & \multicolumn{2}{|c|}{ CLABSI } & \multicolumn{2}{|c|}{ CAUTI } & \multicolumn{2}{|c|}{ SSI } & \multicolumn{2}{|c|}{ Combined } \\
\hline & No. tested & $\% R$ & No. tested & $\% \mathrm{R}$ & No. tested & $\% \mathrm{R}$ & No. tested & $\% R$ \\
\hline \multicolumn{9}{|l|}{ Short-term acute care hospital } \\
\hline Methicillin resistant Staphylococcus aureus & 2,556 & 47.3 & 629 & 49.1 & 3,212 & 44.4 & 6,397 & 46.0 \\
\hline Vancomycin-resistant enterococci & 3,079 & 44.6 & 4,690 & 21.7 & 3,427 & 18.3 & 11,196 & 27.0 \\
\hline ESBL-phenotype Enterobacteriaceae & 2,804 & 21.1 & 11,146 & 16 & 4,184 & 12.6 & 18,134 & 16.0 \\
\hline Carbapenem-resistant Enterobacteriaceae & 3,199 & 4.9 & 10,530 & 2.8 & 4,441 & 1.3 & 18,170 & 2.8 \\
\hline Multidrug-resistant Pseudomonas aeruginosa & 810 & 15.7 & 3,392 & 13.9 & 1,061 & 6.5 & 5,263 & 12.6 \\
\hline Multidrug-resistant Acinetobacter spp. & 369 & 36.6 & 171 & 63.2 & 63 & 47.6 & 603 & 45.3 \\
\hline \multicolumn{9}{|l|}{ Long-term acute care hospital } \\
\hline Methicillin-resistant Staphylococcus aureus & 345 & 75.7 & 50 & 82 & & & 395 & 76.5 \\
\hline Vancomycin-resistant enterococci & 708 & 42.5 & 642 & 62.1 & & & 1,350 & 51.9 \\
\hline ESBL-phenotype Enterobacteriaceae & 401 & 39.7 & 1,324 & 38.2 & & & 1,725 & 38.6 \\
\hline Carbapenem-resistant Enterobacteriaceae & 480 & 14.6 & 1,328 & 11.1 & & & 1,808 & 12.0 \\
\hline Multidrug-resistant $P$ seudomonas aeruiginosa & 138 & 31.9 & 934 & 32.9 & & & 1,072 & 32.7 \\
\hline Multidrug-resistant Acinetobacter spp. & 90 & 73.3 & 80 & 87.5 & & & 170 & 80.0 \\
\hline \multicolumn{9}{|l|}{ Inpatient rehabilitation facility } \\
\hline Methicillin-resistant Staphylococcus aureus & & & 46 & 58.7 & & & 46 & 58.7 \\
\hline Vancomycin-resistant enterococci & & & 190 & 22.6 & & & 190 & 22.6 \\
\hline ESBL-phenotype Enterobacteriaceae & & & 633 & 10.7 & & & 633 & 10.7 \\
\hline Carbapenem-resistant Enterobacteriaceae & & & 634 & 1.9 & & & 634 & 1.9 \\
\hline Multidrug-resistant $P$ seudomonas aeruiginosa & & & 218 & 12.8 & & & 218 & 12.8 \\
\hline Multidrug-resistant Acinetobacter spp. & & & 14 & $-{ }^{\dagger}$ & & & 14 & $-^{\dagger}$ \\
\hline
\end{tabular}

Abbreviations: $\% \mathrm{R}=\%$ resistant to antibiotics; CAUTI $=$ catheter-associated urinary tract infection; CLABSI = central line-associated bloodstream infection; $\mathrm{ESBL}=$ extended-spectrum beta-lactamase; SSI = surgical site infection.

* Empty cells indicate no reporting occurred for that HAl type.

† Insufficient data; fewer than 20 isolates tested for resistance.

this report, declined $24 \%$ in non-intensive care unit (ICU) settings since baseline.* In long-term acute care hospitals, both CLABSIs and CAUTIs have decreased as have CAUTIs in inpatient rehabilitation facilities. The importance of preventing CAUTIs in all settings is highlighted by the frequency with which vancomycin-resistant enterococci, extendedspectrum beta-lactamase phenotype Enterobacteriaceae, and (especially in long-term acute care hospitals) carbapenemresistant Enterobacteriaceae, cause these infections (Table). Collaboration across the U.S. Department of Health and Human Services (HHS), including CDC, the Office of the Assistant Secretary of Health, the Centers for Medicare and Medicaid Services, and the Agency for Health Research and Quality has been important in achieving this success. For example, Centers for Medicare and Medicaid Services reporting and payment incentives have led to greater transparency and accountability, and their Hospital Engagement and Quality Innovation Networks have promoted best practices.

C. difficile has been recently recognized as the most common HAI pathogen in acute care hospitals (4). In 2011, it caused an overall total of 453,000 infections, and 29,000 patients died within 30 days of diagnosis (16); 94\% of all CDIs are related to various precedent or concurrent health care exposures (17). The CDI SIR in acute care hospitals decreased only $8 \%$ overall during 2011-2014, and more concerning, increased $4 \%$ during 2013-2014. More work is needed to ensure that patients are safe from $C$. difficile and AR bacteria.

Controlling AR threats is linked to preventing the occurrence of HAIs, reducing selective pressure by improving overall antibiotic stewardship, and preventing the spread of AR bacteria within and between facilities. Preventing catheter- and procedure-related infections can be accomplished by always following recommended indications and guidelines for insertion, maintenance, and removal of vascular and bladder catheters. CDC and its partners are implementing new HHS-proposed HAI targets for December 2020, using 2015 NHSN data as its new baseline. A key strategy for reaching these goals is the Targeted Assessment for Prevention strategy to identify gaps in infection control in facilities with a disproportionate number of HAIs (18). In addition to reducing the need for antibiotics used in treatment, preventing HAIs prevents complications of infection, including sepsis, a major cause of death

\footnotetext{
*http://www.cdc.gov/hai/progress-report/index.html.
} 


\section{Key Points}

- Antibiotic-resistant (AR) bacteria can make infections impossible to treat, especially given the extensive resistance frequently encountered in health care facilities. Of 18 AR bacteria identified by CDC as public health threats, six, in addition to Clostridium difficile, cause health care-associated infections (HAIs).

- Three common HAIs associated with catheters placed in a vein or the bladder and procedures (operations) include: central-line associated blood stream infections (CLABSIs), catheter-associated urinary tract infections (CAUTIs), and surgical site infections (SSIs).

- Preventing these HAIs is an important strategy for reducing the impact of AR bacteria on human health, including the prevention of sepsis and death. Considerable progress has been made for some but not all HAIs. Compared with baseline historic data from 5-8 years earlier, CLABSIs decreased by $50 \%$ and SSIs by $17 \%$ in 2014 . Whereas CAUTIs appear unchanged from baseline, there have been recent decreases. $C$. difficile infections in hospitals decreased 8\% during 2011-2014.

- In 2014, the chance that an HAI was caused by one of the six AR threat bacteria was one in seven in shortterm acute care hospitals but higher in other health care settings such as long-term acute care hospitals where it was one in four.

- Physicians, nurses, and health care leaders, working together with the help of CDC, other federal agencies, and other partners, need to consistently combine strategies to prevent catheter- and procedure-related HAIs, prevent the spread of AR bacteria, and improve antibiotic use, thereby preventing further patient harm caused by AR HAIs.

- Additional information available at http://www.cdc. gov/vitalsigns.

In conjunction with HAI prevention is implementation of hospital antibiotic stewardship programs (19). This is accomplished by always obtaining cultures when starting necessary antibiotics and, especially in septic patients, doing so promptly, using culture results to reassess the continued need for antibiotics, discontinuing antibiotics that are no longer needed or to which AR has developed, and using the appropriate drug at the proper dose and administration frequency. Antibiotic exposure is well recognized as the most important modifiable risk factor for CDI, and antibiotic stewardship is potentially the most effective CDI prevention strategy $(17,19)$. The emergence and spread of the hypervirulent, fluoroquinoloneresistant, ribotype 027 strain of $C$. difficile in North America and Europe was facilitated by increased use of fluoroquinolones (20). Reducing unnecessary use of this antibiotic class has been instrumental in facilities where control of this strain has been achieved $(21,22)$. What is less widely recognized is the role that disruption of the human microbiome has on increasing patients' risk for acquiring AR strains of other HAI pathogens (23) and, once colonized, developing infection (24). Genes that confer resistance can be carried on the same plasmid or chromosome as genes that increase bacterial virulence, leading to the emergence of highly adapted AR HAI pathogens (25). CDC summarizes core elements of successful stewardship programs, which can help assure the prompt initiation of necessary antibiotics and reduce unnecessary antibiotic use, thereby reducing the risk of $\mathrm{CDI}$ and $\mathrm{AR}$ infections, improving individual patient outcomes, and saving health care dollars (19).

In conjunction with HAI prevention and antibiotic stewardship, the third necessary strategy is the prevention of cross transmission. To achieve this, physicians, nurses, and health care leaders need to improve hand hygiene, room cleaning, and use of personal protective equipment, and be aware of HAI outbreaks caused by AR bacteria in their hospital or region. In the case of $C$. difficile, which is unique among the AR threat bacteria in forming spores, special environmental measures might also be needed to prevent transmission (17). Because AR strains might be more virulent than other strains and thereby more likely to colonize and infect patients already receiving antibiotics, interrupting transmission of these strains reduces both the number of HAIs and the likelihood that an HAI is caused by an AR threat. To assist clinicians, health care leaders, and state and local public health authorities to learn when well-adapted resistant strains are emerging and spreading in a region, $\mathrm{CDC}$ is working with partners to build networks to better detect and respond to AR threats and to make antibiotic resistance data from health care facilities more readily accessible through a new HAI Antibiotic Resistance Patient Safety Atlas. ${ }^{\dagger}$

Over one of every four HAIs reported from long-term acute care hospitals were caused by AR bacteria. Moreover, limited data suggest CDI incidence in long-term acute care hospitals might be several fold higher than in short-term acute care hospitals $(26,27)$. One contributing factor is patient transfer from intensive care units of acute care hospitals where their microbiomes have been disrupted by exposure to antibiotics and where they have been colonized with AR threat bacteria (28). Long-term acute care hospitals are facilities that can transmit or amplify antibiotic resistance within a community

\footnotetext{
${ }^{\dagger}$ http://www.cdc.gov/hai/surveillance/ar-patient-safety-atlas.html.
} 
of interconnected health care facilities (29). It is critical for interconnected health care facilities to work together for early detection and response to emerging AR threats; coordinated prevention initiatives have the biggest impact on a community or region overall (30). Through sharing of information, practical expertise, and regional leadership, coordinated activity can have a larger impact on preventing transmission and infections with antibiotic-resistant bacteria than hospitals working alone.

The findings in this report are subject to at least two limitations. First, infections included in SIR calculations were a subset of all the infections evaluated for AR. The latter included infection events reported from any type of SSI, and infections occurring in locations regardless of eligibility to calculate a SIR. Second, the reported resistance relied on the manual reporting of the facility staff, based on reports provided by the clinical laboratory, and might contain inaccurate test results, data entry errors, and some incomplete information. Despite these limitations, these data provide important information on the status of HAI infection prevention in the United States in 2014 and the persistent challenge of preventing the spread of AR bacteria in a variety of inpatient health care settings. Preventing HAIs and the spread of antibiotic resistance is possible if physicians, nurses, and health care leaders consistently and comprehensively follow all recommendations to prevent HAIs, including prevention of catheter- and procedure-related infections, antimicrobial stewardship, and implementation of measures to prevent spread.

${ }^{1}$ Division of Healthcare Quality Promotion, National Center for Emerging and Zoonotic Infectious Diseases, CDC.

Corresponding author: L. Clifford McDonald, ljm3@cdc.gov, 404-639-3833.

\section{References}

1. CDC. Antibiotic resistance threats in the United States. Atlanta, GA: US Department of Health and Human Services, CDC; 2013. http://www. cdc.gov/drugresistance/threat-report-2013/

2. White House. National action plan to combat antibiotic-resistant bacteria. Washington DC: White House; 2015. https:/www.whitehouse.gov/sites/ default/files/docs/national_action_plan_for_combating_antiboticresistant_bacteria.pdf

3. Magill SS, Edwards JR, Beldavs ZG, et al. Prevalence of antimicrobial use in US acute care hospitals, May-September 2011. JAMA 2014;312:143846. http://dx.doi.org/10.1001/jama.2014.12923

4. Magill SS, Edwards JR, Bamberg W, et al. Multistate point-prevalence survey of health care-associated infections. N Engl J Med 2014;370:1198208. http://dx.doi.org/10.1056/NEJMoa1306801

5. US Department of Health and Human Services. National action plan to reduce healthcare-associated infections. Washington DC: US Department of Health and Human Services; 2010. http://www.hhs.gov/ash/initiatives/ hai/actionplan/index.html

6. CDC. National Healthcare Safety Network: acute care hospital surveillance for central line-associated bloodstream infections. Atlanta, GA: US Department of Health and Human Services, CDC; 2016. http:// www.cdc.gov/nhsn/acute-care-hospital/clabsi/index.html
7. CDC. National Healthcare Safety Network: acute care hospital surveillance for catheter-associated urinary tract infections. Atlanta, GA: US Department of Health and Human Services, CDC; 2016. http:// www.cdc.gov/nhsn/acute-care-hospital/cauti/index.html

8. CDC. National Healthcare Safety Network: acute hospital surveillance for surgical site infections. Atlanta, GA: US Department of Health and Human Services, CDC; 2015. http://www.cdc.gov/nhsn/acute-carehospital/ssi/index.html

9. CDC. National and state healthcare associated infections progress report (2012). Atlanta, GA: US Department of Health and Human Services, CDC; 2014. http://www.cdc.gov/HAI/pdfs/progress-report/ hai-progress-report-2014.pdf

10. CDC. National and state healthcare associated infections progress report (2013). Atlanta, GA: US Department of Health and Human Services, CDC; 2015. http://www.cdc.gov/hai/surveillance/nhsn_nationalreports.html

11. Mu Y, Edwards JR, Horan TC, Berrios-Torres SI, Fridkin SK. Improving risk-adjusted measures of surgical site infection for the national healthcare safety network. Infect Control Hosp Epidemiol 2011;32:970-86. http:// dx.doi.org/10.1086/662016

12. CLSI. Performance standards for antimicrobial susceptibility testing; 23rd informational supplement. In: CaLSI, editor. CLSI document M100-S23. Wayne, PA: CLSI; 2013.

13. Council of State and Territorial Epidemiologists. Standardized definition for carbapenem-resistant Enterobacteriaceae (CRE) and recommendation for sub-classification and stratified reporting. http://c.ymcdn.com/sites/ www.cste.org/resource/resmgr/2015PS/2015PSFinal/15-ID-05.pdf

14. CDC. Vital signs: central line-associated blood stream infectionsUnited States, 2001, 2008, and 2009. MMWR Morb Mortal Wkly Rep 2011;60:243-8.

15. Scott RD 2nd, Sinkowitz-Cochran R, Wise ME, et al. CDC centralline bloodstream infection prevention efforts produced net benefits of at least \$640 Million during 1990-2008. Health Aff (Millwood) 2014;33:1040-7. http://dx.doi.org/10.1377/hlthaff.2013.0865

16. Lessa FC, Mu Y, Bamberg WM, et al. Burden of Clostridium difficile infection in the United States. N Engl J Med 2015;372:825-34. http:// dx.doi.org/10.1056/NEJMoa1408913

17. CDC. Vital signs: preventing Clostridium difficile infections. MMWR Morb Mortal Wkly Rep 2012;61:157-62.

18. CDC. The five "W"s of the targeted assessment for prevention (TAP) strategy. Atlanta, GA: US Department of Health and Human Services, CDC; 2015. http://www.cdc.gov/hai/prevent/tap.html

19. Fridkin S, Baggs J, Fagan R, et al. Vital signs: improving antibiotic use among hospitalized patients. MMWR Morb Mortal Wkly Rep 2014;63:194-200.

20. McDonald LC, Killgore GE, Thompson A, et al. An epidemic, toxin gene-variant strain of Clostridium difficile. N Engl J Med 2005;353:2433-41. http://dx.doi.org/10.1056/NEJMoa051590

21. Kallen AJ, Thompson A, Ristaino P, et al. Complete restriction of fluoroquinolone use to control an outbreak of Clostridium difficile infection at a community hospital. Infect Control Hosp Epidemiol 2009;30:264-72. http://dx.doi.org/10.1086/595694

22. Wilcox MH, Shetty N, Fawley WN, et al. Changing epidemiology of Clostridium difficile infection following the introduction of a national ribotyping-based surveillance scheme in England. Clin Infect Dis 2012;55:1056-63. http://dx.doi.org/10.1093/cid/cis614

23. Swaminathan M, Sharma S, Poliansky Blash S, et al. Prevalence and risk factors for acquisition of carbapenem-resistant Enterobacteriaceae in the setting of endemicity. Infect Control Hosp Epidemiol 2013;34:809-17. http://dx.doi.org/10.1086/671270

24. Borer A, Saidel-Odes L, Eskira S, et al. Risk factors for developing clinical infection with carbapenem-resistant Klebsiella pneumoniae in hospital patients initially only colonized with carbapenem-resistant K. pneumoniae. Am J Infect Control 2012;40:421-5. http://dx.doi. org/10.1016/j.ajic.2011.05.022 
25. Beceiro A, Tomás M, Bou G. Antimicrobial resistance and virulence: a successful or deleterious association in the bacterial world? Clin Microbiol Rev 2013;26:185-230. http://dx.doi.org/10.1128/CMR.00059-12

26. Brakovich B, Bonham E, VanBrackle L. War on the spore: Clostridium difficile disease among patients in a long-term acute care hospital. J Healthc Qual 2013;35:15-21. http://dx.doi. org/10.1111/j.1945-1474.2011.00182.x

27. Goldstein EJ, Polonsky J, Touzani M, Citron DM. C. difficile infection (CDI) in a long-term acute care facility (LTAC). Anaerobe 2009;15:241-3. http://dx.doi.org/10.1016/j.anaerobe.2009.06.009
28. Halpin AL, de Man T, Kraft C, et al. Intestinal microbiome disruption in patients in a long-term acute care hospital: a case for development of microbiome disruption indices to improve infection prevention. Am J Infect Control 2016. Available ahead of print. http://www.ajicjournal. org/article/S0196-6553(16)00040-7/fulltext

29. Lin MY, Lyles-Banks RD, Lolans K, et al. The importance of longterm acute care hospitals in the regional epidemiology of Klebsiella pneumoniae carbapenemase-producing Enterobacteriaceae. Clin Infect Dis 2013;57:1246-52 http://dx.doi.org/10.1093/cid/cit500

30. Slayton RB, Toth D, Lee BY, et al. Vital signs: Estimated effects of a coordinated approach for action to reduce antibiotic-resistant infections in health care facilities - United States. MMWR Morb Mortal Wkly Rep 2015;64:826-31. http://dx.doi.org/10.15585/mmwr.mm6430a4 